\title{
Mechanical Tissue Compression and Whole-mount Imaging at Single Cell Resolution for Developing Murine Epididymal Tubules
}

Tsuyoshi Hirashima ${ }^{1,2, *}$

1Department of Pathology and Biology of Diseases, Graduate School of Medicine, Kyoto University, Kyoto, Japan; 2Japan Science and Technology Agency, PRESTO, Sakyo-ku, Kyoto, Japan

*For correspondence: hirashima.tsuyoshi.2m@kyoto-u.ac.jp

\begin{abstract}
[Abstract] Cells inside the body are subjected to various mechanical stress, such as stretch or compression provided by surrounding cells, shear stresses by blood or lymph flows, and normal stresses by luminal liquids. Force loading to the biological tissues is a fundamental method to better understand cellular responses to such mechanical stimuli. There have been many studies on compression or stretch experiments that target culture cells attached to a flexible extensible material including polydimethylsiloxane (PDMS); however, the know-how of those targeting to tissues is still incomplete. Here we present the protocol for mechanical tissue compression and image-based analysis by focusing on developing murine epididymis as an example. We show a series of steps including tissue dissection from murine embryos, hydrogel-based compression method using a manual device, and non-destructive volumetric tissue imaging. This protocol is useful for quantifying and exploring the biological mechanoresponse system at tissue level.
\end{abstract}

Keywords: Epididymal tube, Mechanical stimuli, Optical clearing, Tissue compression, Whole-mount imaging

[Background] Cells can respond to mechanical stimuli through intracellular biochemical signaling pathways. This cellular mechanoresponse is known to play a fundamental role in various biological processes such as embryonic development, regeneration, tissue homeostasis, and cancer metastasis (Mammoto et al., 2013; Humphrey et al., 2014; Vining and Mooney, 2017). Recent experiments regarding force loading externally to the cells have revealed how cells react to the given mechanical stimuli. For example, it has been shown that stretching or compressing a cell monolayer attaching to a flexible silicone substrate like polydimethylsiloxane (PDMS) triggers a mechanosensitive ion channel protein Piezo1 to evoke various cellular behaviors such as cell division and extrusion, leading to homeostatic cell number in the tissues (Eisenhoffer et al., 2012; Gudipaty et al., 2017). There are various force manipulations to adherent culture cells; however, the manipulation methods to volumetric tissues are far from sufficient (Campàs, 2016). Force manipulation can be performed using magnetic oil droplets but specialized devices are required (Serwane et al., 2016). Here we show an easier way to compress a tubular tissue and examine the cellular response by specifically focusing on the murine epididymis. 


\section{Materials and Reagents}

1. $35 \mathrm{~mm}$ CELLview ${ }^{\mathrm{TM}}$ dish with glass bottom (Greiner, catalog number: 627871)

2. $2 \mathrm{ml}$ round-bottom microtube (Watson, catalog number: 132-620C)

3. Institute of Cancer Research (ICR) pregnant mice (Japan SLC, Slc:ICR)

4. Dulbecco's modified Eagle's medium (DMEM) (4.5 g/L Glucose) without phenol red (Nacalai Tesque, catalog number: 08489-45)

5. Fetal bovine serum (FBS) (Thermo Fisher Scientific, catalog number: 12483)

6. Penicillin-streptomycin mixed solution (Nacalai Tesque, catalog number: 26253-84)

7. Hank's balanced salt solution (HBSS) with $\mathrm{Ca}, \mathrm{Mg}$, without phenol red (Nacalai Tesque, catalog number: 09735-75)

8. Triton X-100 (Nacalai Tesque, catalog number: 35501-15)

9. 4\%-Paraformaldehyde (PFA) phosphate buffer solution (Nacalai Tesque, catalog number: 09154-85)

10. Normal goat serum (Abcam, catalog number: ab156046)

11. Rabbit monoclonal anti-E-cadherin (24E10) antibody (Cell Signaling Technology, catalog number: 3195)

12. Goat anti-rabbit IgG $(\mathrm{H}+\mathrm{L})$ highly cross-adsorbed secondary antibody, Alexa Fluor 546 conjugate (Thermo Fisher Scientific, catalog number: A-11035)

13. Agarose (Nippon Gene, catalog number: 316-01191)

14. Cellmatrix collagen type I-A (3 mg/ml, pH 3.0) (Nitta Gelatin, catalog number: 637-00653)

15. Matrigel growth factor reduced (Corning, catalog number: 356231 )

16. Tissue clearing reagents CUBIC-R+ (Tokyo Chemical Industry, catalog number: T3741)

17. Culture Media (see Recipes)

18. PBST (see Recipes)

\section{Equipment}

1. Forceps (Fine Science Tools, catalog number: 11251-30)

2. Scissors with straight and sharp tip (Fine Science Tools, catalog number: 14084-08)

3. Polydimethylsiloxane (PDMS) stretch chamber (STREX, catalog number: STB-CH-04)

4. Manual uniaxial stretching device (STREX, catalog number: STB-100)

5. Plasma cleaning equipment (STREX, catalog number: PC-40)

6. Tube rotator (WakenBtech, model: WKN-2210)

7. $\mathrm{CO}_{2}$ incubator (Sanyo, model: MCO-18AIC)

8. Shaker (WakenBtech, model: WKN-2240)

9. Confocal microscope (Leica, model: TCS SP8) 


\section{Software}

1. ImageJ (National Institute of Health, https://imagej.nih.gov/ij/)

\section{Procedure}

A. Tissue dissection

1. Sacrifice a mouse at the desired day of gestation according to all relevant international and institutional regulations relating to animal acquirement, care, and use.

Notes:

a. We routinely use ICR mice but any mouse strain can be used.

b. For epididymis compression, embryonic day $(E) 14.5$ or E15.5 is suitable because the epididymal tubule is relatively straight and thereby compression direction is appropriately determined.

2. Collect embryos carefully using the scissor and forceps, and excise epididymis with testis in the HBSS buffer at room temperature. Please see Video 1 for details.

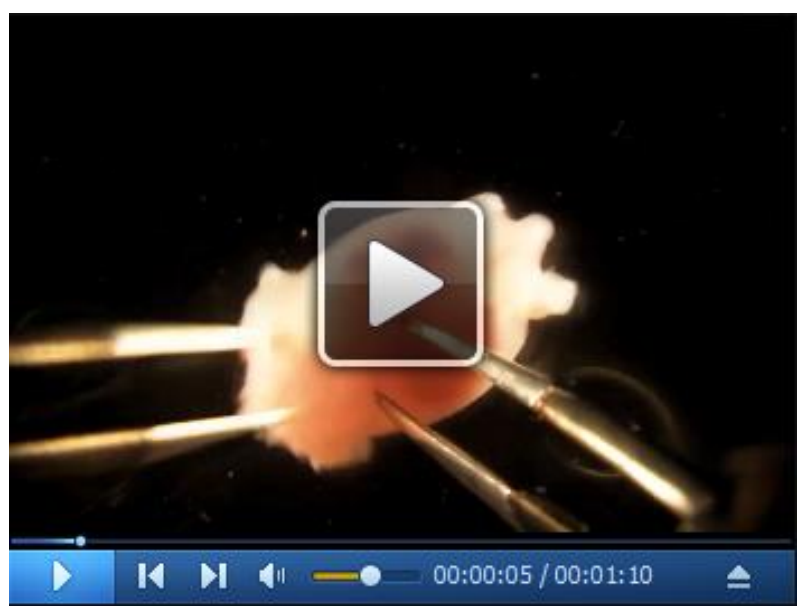

Video 1. Dissection of epididymis from embryos at E15.5. The four limbs, the tail, and the upper tissue above the neck were removed. The embryo lies down on its face and its anterior is facing left.

3. Remove the testis from the excised tissue and isolate epididymis (Figure 1). 


\section{bḯ-protocol

A

B
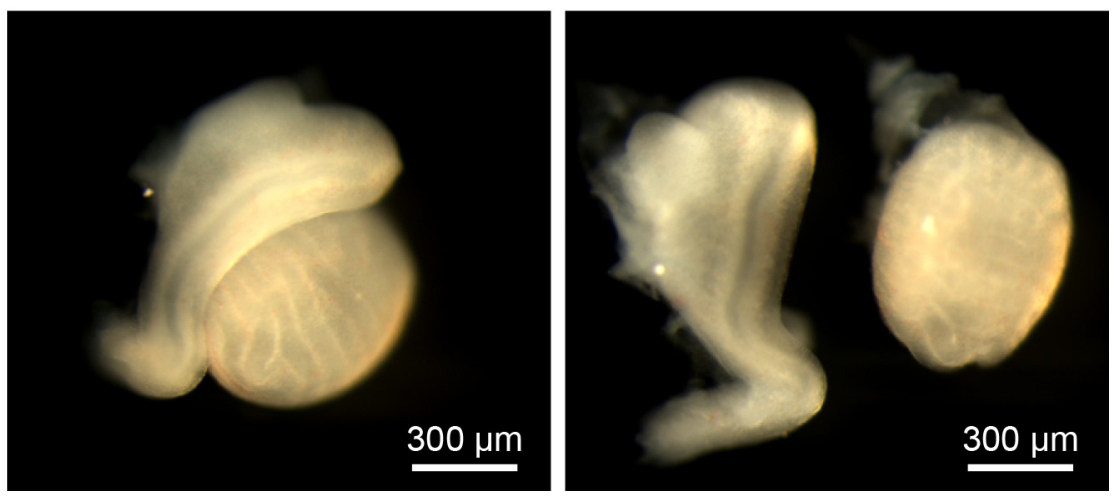

Figure 1. Separation of epididymis and testis. A. Epididymis with testis dissected at E15.5 before separation. B. After separation. Left is epididymis and right is testis. Scale bars: $300 \mu \mathrm{m}$.

4. Incubate the isolated epididymides with HBSS in a dish on ice.

Note: The samples should be used for the tissue compression within $1 \mathrm{~h}$.

B. Tissue compression

Note: This experiment can be performed outside of a tissue culture hood.

1. Hydrophilize the PDMS stretch chamber using the plasma cleaning equipment for $10 \mathrm{~s}$.

Note: This process enhances the adhesion between the chamber and hydrogels into which the epididymides are embedded but not required.

2. Inset the PDMS stretch chamber in the uniaxial stretching device (Figure 2A), and stretch the chamber using the device manually according to the manufacturer recommendations (Figure 2B).

A

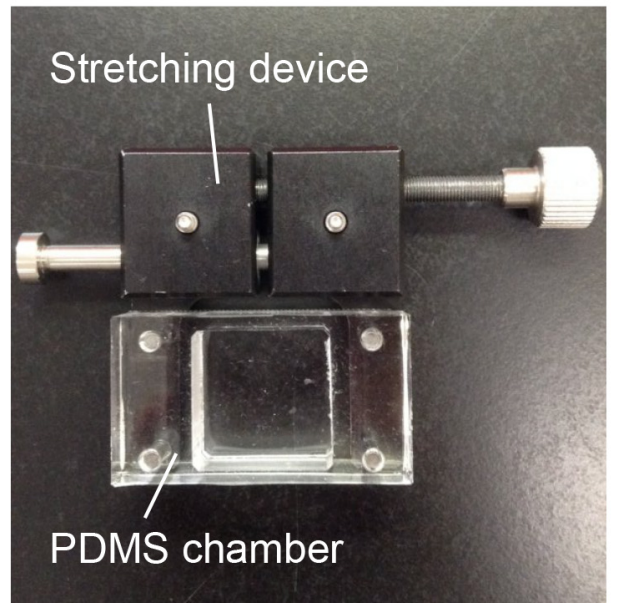

B

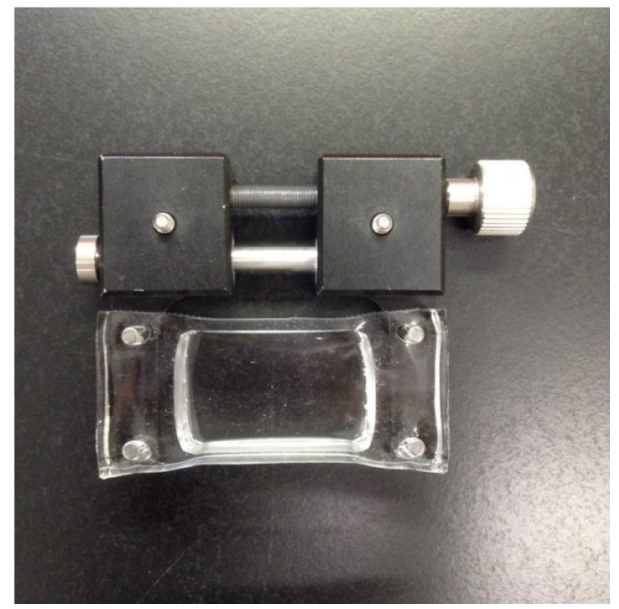

Figure 2. Manual uniaxial stretching device with PDMS chamber. A. Before stretch. B. After stretch. 
3. Transfer the isolated epididymides to the inside of the chamber and remove traces of the HBSS buffer around the samples as much as possible by pipetting.

4. Fill the chamber with $1 \mathrm{ml}$ collagen gels or Matrigel without any dilution.

Notes:

a. Regarding the hydrogels, either collagen gel or Matrigel is fine although the chemical stimuli to the biological tissues depending on a type of gels have been unknown.

b. The hydrogels should be pre-thawed at $4{ }^{\circ} \mathrm{C}$ and keep the temperature right up to the insertion of tissues according to the manufacturer recommendations.

5. Align the orientation of epididymis using the forceps according to the target direction of compression (Figure 3).

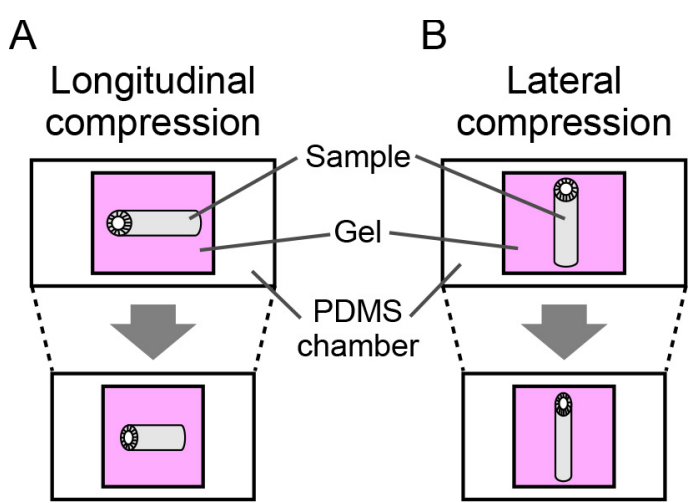

Figure 3. Orientation of tissue compression. The longitudinal axis of the epididymis is aligned along the direction of compression. A. Longitudinal compression. B. Lateral compression.

6. Incubate at $37^{\circ} \mathrm{C}$ for $10 \mathrm{~min}$ for gelation.

7. Add $1 \mathrm{ml}$ of the culture medium (Recipe 1) prewarmed at $37^{\circ} \mathrm{C}$ onto the gels inside of the chamber.

8. Release the chamber from the stretched state by turning the screw of the stretching device at a constant speed, e.g., $\sim 0.2 \mathrm{~mm} / \mathrm{s}$.

Notes:

a. Remember setting the negative control. One is no compressive treatment.

b. For large compression (e.g., $33 \%$ ), tissue compression can be visually confirmed.

9. Incubate at $37^{\circ} \mathrm{C}$ under $5 \% \mathrm{CO}_{2}$ until the timing for the analysis.

Note: 10 min compression led to tissue-scale deformation, but not cell rearrangement. However, longer compression resulted in cell rearrangement perhaps due to active cell force generation by the external mechanical stimulation.

C. Whole-mount staining of E-cadherin, optical clearing, and imaging

1. Fix the samples by adding $2 \mathrm{ml}$ of $4 \%$ PFA into the chamber and incubate at $4{ }^{\circ} \mathrm{C}$ overnight.

2. Take out the epididymides from the gels using the forces, and transfer them to the $2 \mathrm{ml}$ microtube containing $1 \mathrm{ml}$ PBST (Recipe 2). The following processes and reactions are made in the 
microtube. Aspirate the PBST by pipetting.

3. Add $200 \mu \mathrm{l}$ of $10 \%$ normal goat serum in PBST and incubate at $37^{\circ} \mathrm{C}$ for $3 \mathrm{~h}$ for blocking.

4. Gently wash the samples twice. Every one of the two washes in PBST should last 5 min with the rotator at room temperature.

5. Add $50 \mu \mathrm{l}$ of the anti-E-Cadherin antibody (primary antibody) diluted in PBST (1:200) and incubate at $4{ }^{\circ} \mathrm{C}$ overnight.

6. Gently wash the samples three times and every one of the three washes in PBST should last $1 \mathrm{~h}$ with the rotator at room temperature.

7. Add $50 \mu \mathrm{l}$ of the anti-rabbit conjugated with Alexa Fluor 546 (secondary antibody) diluted in PBST $(1: 1,000)$ and incubate at $4{ }^{\circ} \mathrm{C}$ overnight or at room temperature for $3 \mathrm{~h}$.

Note: Use a longer wavelength of conjugated fluorescence dye for volumetric tissue imaging.

8. Gently wash the samples $3 \mathrm{x}$ in PBST with the rotator at room temperature at $1 \mathrm{~h}$ intervals.

9. Transfer the samples onto the glass base dish and remove surrounding liquids by pipetting.

10. Drop $5 \mu \mathrm{l}$ of $1 \%$ agarose in PBS melted at $80^{\circ} \mathrm{C}$ to the samples and spread the mix to form a gel and thus stick the samples and glass for physical fixation.

11. Cover the samples with PBS after agarose gelation.

12. Optical clearing

a. Aspirate the PBS, add the premixed optical clearing reagents CUBIC-R+ in PBS (1:1), and incubate at $37^{\circ} \mathrm{C}$ for 30 min with gentle shaking using the shaker.

b. Aspirate the CUBIC-R+/PBS, add the CUBIC-R+ solution, and incubate at $37^{\circ} \mathrm{C}$ for $2 \mathrm{~h}$ with gentle shaking.

Note: Choose clearing solutions according to your samples and purpose. Please see Richardson and Lichtman (2015), Hirashima and Adachi (2015), and Susaki and Ueda (2016) for the choice of solutions.

13. Acquire stack images under a confocal microscope and open them using ImageJ. Figure 4 shows images of the stained E-cadherin in epididymides compressed for $10 \mathrm{~min}$ in different directions as examples. Use 'Image $\rightarrow$ Stacks $\rightarrow$ Orthogonal Views' of ImageJ for visualization in different cross-sections. 


\section{b̆̈̈-protocol

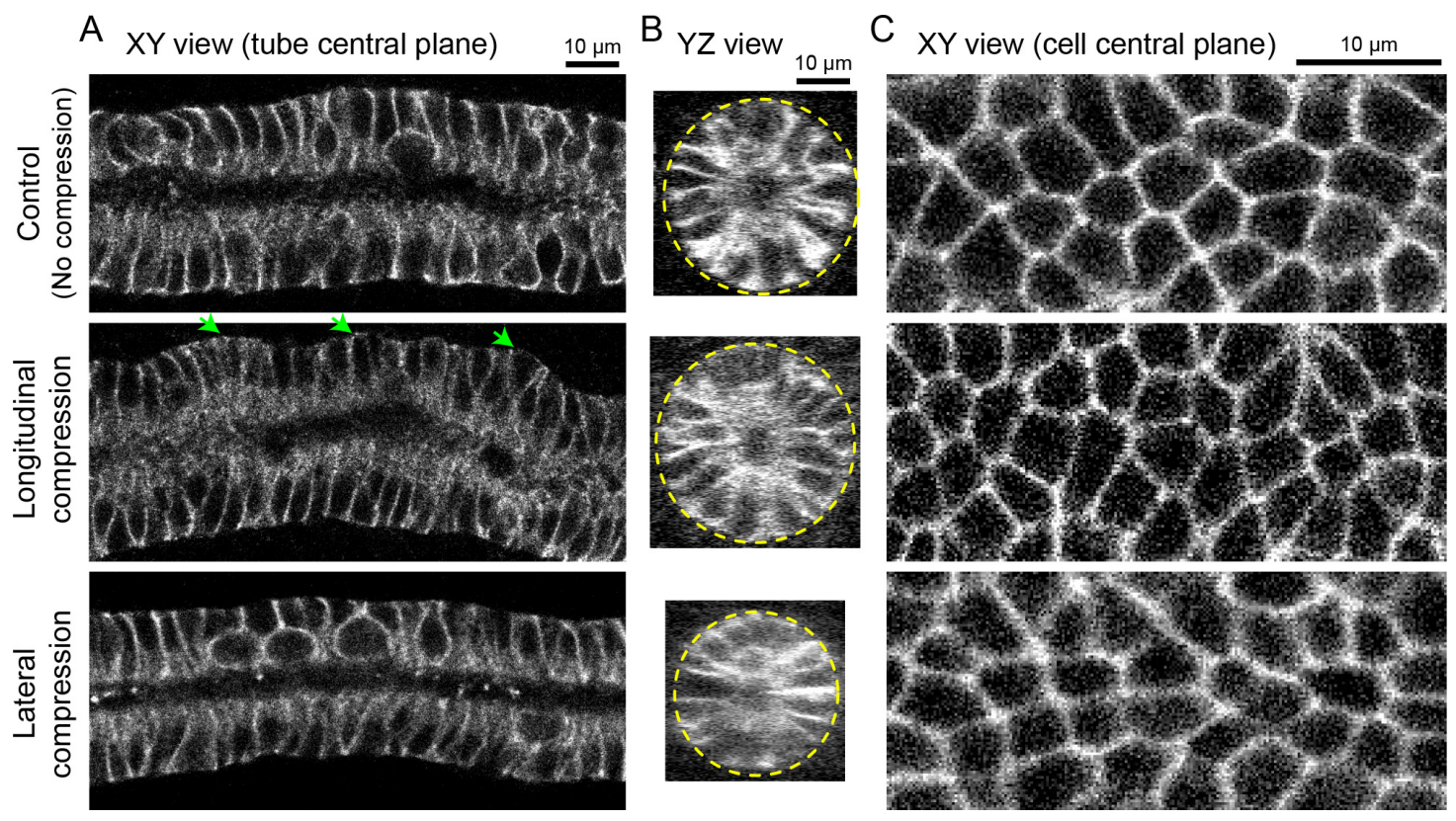

Figure 4. Tissue and cell morphology in compressed epididymides. The epithelial cells were stained with E-cadherin antibody. The samples were compressed $~ 33 \%$ (release from $150 \%$ stretch state to $100 \%$ ) for $10 \mathrm{~min}$. Upper row: control (no compression), middle row: longitudinal compression, lower row: lateral compression. A. XY view at the central plane of the tube. Note that the epididymal tubule was buckled due to the longitudinal compression at the tissue scale (green arrows). Scale bar $=10 \mu \mathrm{m}$. B. YZ view at a cross-section of the tube. Yellow dotted lines represent the periphery of the tube. Lateral compression makes the tube thin isotropically while longitudinal compression makes it slightly thick in the buckled point. Scale bar $=10 \mu \mathrm{m}$. C. XY view at a central plane of epithelial cells (middle point of apico-basal axis). Note that cell shape deforms according to the direction of the uniaxial compression. Scale bar $=10 \mu \mathrm{m}$.

\section{$\underline{\text { Notes }}$}

This protocol focuses on tissue compression and image-based analysis for developing epididymis.

However, we confirmed that this protocol can apply to murine mesenchyme-free lung epithelium dissected at least E12.5 to E14.5 and murine trachea dissected at least E12.5. Also, not only compressing but also stretching the tissues can be achieved with slight modifications, e.g., skipping the step of releasing the device from the prestretch state.

\section{$\underline{\text { Recipes }}$}

1. Culture Media

DMEM containing 10\% FBS and 1\% penicillin-streptomycin mixed solution

2. PBST

$0.1 \%$ Triton $X-100$ in PBS 


\section{Acknowledgments}

I would like to thank Yu Kurata for technical assistants and Taiji Adachi for the use of the facilities in the lab. This work was supported by the JSPS KAKENHI grant 17 KT0107 and by the JST PRESTO grant JPMJPR1949. This protocol corresponds to Hirashima and Adachi (2019).

\section{Ethics}

All the animal experiments were approved by the local ethical committee for animal experimentation (MedKyo 19090) and were performed in compliance with the guide for the care and use of laboratory animals at Kyoto University.

\section{Competing interests}

The author declares no competing interests.

\section{References}

1. Campàs, O. (2016). A toolbox to explore the mechanics of living embryonic tissues. Semin Cell Dev Biol 55: 119-130.

2. Eisenhoffer, G. T., Loftus, P. D., Yoshigi, M., Otsuna, H., Chien, C. B., Morcos, P. A. and Rosenblatt, J. (2012). Crowding induces live cell extrusion to maintain homeostatic cell numbers in epithelia. Nature 484(7395): 546-549.

3. Gudipaty, S. A., Lindblom, J., Loftus, P. D., Redd, M. J., Edes, K., Davey, C. F., Krishnegowda, V. and Rosenblatt, J. (2017). Mechanical stretch triggers rapid epithelial cell division through Piezo1. Nature 543(7643): 118-121.

4. Hirashima, T. and Adachi, T. (2015). Procedures for the quantification of whole-tissue immunofluorescence images obtained at single-cell resolution during murine tubular organ development. PLoS One 10(8): e0135343.

5. Hirashima, T. and Adachi, T. (2019). Polarized cellular mechano-response system for maintaining radial size in developing epithelial tubes. Development 146(23).

6. Humphrey, J. D., Dufresne, E. R. and Schwartz, M. A. (2014). Mechanotransduction and extracellular matrix homeostasis. Nat Rev Mol Cell Biol 15(12): 802-812.

7. Mammoto, T., Mammoto, A. and Ingber, D. E. (2013). Mechanobiology and developmental control. Annu Rev Cell Dev Biol 29: 27-61.

8. Richardson, D. S. and Lichtman, J. W. (2015). Clarifying tissue clearing. Cell 162(2): 246-257.

9. Serwane, F., Mongera, A., Rowghanian, P., Kealhofer, D. A., Lucio, A. A., Hockenbery, Z. M. and Campas, O. (2017). In vivo quantification of spatially varying mechanical properties in developing tissues. Nat Methods 14(2): 181-186. 
10. Susaki, E. A. and Ueda, H. R. (2016). Whole-body and whole-organ clearing and imaging techniques with single-cell resolution: toward organism-level systems biology in mammals. Cell Chem Biol 23(1): 137-157.

11. Vining, K. H. and Mooney, D. J. (2017). Mechanical forces direct stem cell behaviour in development and regeneration. Nat Rev Mol Cell Biol 18(12): 728-742. 